\title{
Retrospective Clinical Survey: A Study to Show the Effect of Nidra Viparyaya on Dhatu Sarata
}

\author{
Dr. Manisha Sharma ${ }^{1}$, Dr. Bharti ${ }^{2}$, Dr. Subhash Sharma ${ }^{3}$, Dr. Raman Toor ${ }^{4}$ \\ ${ }^{1}$ M.D. Ayu, Reader, Department of Rog Nidan, G.N.A.M.C., Gopalpur, Ludhiana (Pb.) \\ ${ }^{2}$ M.D. Ayu.,Reader, Department of Kayachikitsa, G.N.A.M.C., Gopalpur, Ludhiana (Pb.) \\ ${ }^{3}$ M.D. Ayu., Reader, Department of Panchkarma, G.N.A.M.C., Gopalpur, Ludhiana (Pb.) \\ ${ }^{4}$ M.D. Ayu., Lecturer, Department of Agad Tantra, G.N.A.M.C., Gopalpur, Ludhiana (Pb.) \\ *Corresponding Author: Dr. Manisha Sharma \\ Reader, Department of Rog Nidan, G.N.Ayu.College and Research Institute, Gopalpur (Ludhiana) Pb.
}

\begin{abstract}
Acharya Charaka has designated ahara (food), nidra (sleep) and brahmacharya (abstinence) as the main three pillars of life termed as trayaupastambhas. These, when taken in adequate amount at the right time provide stability to the body and improve bala (stremgth), varna (complexion) and upchaya (metabolism) in the body. Among these, nidra plays a major role in one's life. Adequate sleep leads to arogya (health), bala shukra vridhi (increase in physical and reproductive strength) and dhatusamyata and healthy life which is the ultimate goal of Ayurveda. On the other hand, altered sleep leads to bala hani (loss of strength), rugnavastha (diseased state) or even death. So it can be said that abnormal or inadequate nidra can manifest in the form of dhatuvikara, making a person atura (diseased). For better economic gains, people are neglecting their daily needs of having proper ahara, nidra and other required vihara at proper time. Due to these reasons, people are becoming more prone towards diseased state. Dashavidha-atura pariksha, given by Acharya Charak is very unique and important concept of Ayurveda which is done to know pramana (magnitude) of ayu (life), dosha and bala (strength) of rogi. Dhatusara pariksha is one among these which literally means essence of $d h a t u$, possessing all good qualities in excellent standard without any defects. The present study was done to study the relationship of nidra on dhatusarata, with the purpose that people should understand the importance of nidra as only a healthy individual can lead to the formation of a healthy society.
\end{abstract}

Keywords: Nidra, Trayaupastambhas, Dhatusarata,

\section{INTRODUCTION}

Ayurveda has mentioned the aims as, to maintain the healthy status of a human being and secondly to cure the diseased ones ${ }^{1}$.Various concepts have been mentioned in Ayurvedic Classics to keep oneself healthy which are in the form of various preventive measures like Yoga, Achara Rasayana (well conducted behavior), Dinacharya (daily regimen), Rutucharya (seasonal regimen) etc. One among them is Traupastambha i.e. ahara (food), nidra (sleep) and brahmacharya (abstinence), which when taken in adequate amount at the accurate time provide stability to the body and improve bala (stength), varna (complexion) and upchaya (metabolism) in the body ${ }^{2}$ which directly leads to swasthya (health) and that is 
the ultimate goal of Ayurveda. Sidewise, cause klaibyata (impotence) bala hani (loss of strength), aturavastha (diseased state) or even death if taken inadequately.

Dashavidha-atura pariksha ${ }^{3}$ is also considered as very unique and important concept of Ayurveda which has been mentioned by Acharya Charak in Charak vimanasthana Chapter 8. The purpose of rogi pariksha (patient examination) is to know the pramana (quantity) of ayu (age), dosha and bala (strength) of rogi which is very much essential for the selection of drug, dose adjustment and plan of treatment. Dashavidha-atura pariksha includes-

1) Prakriti pariksha (Examination of basic physical and psychological component)

2) Vikriti pariksha (Examination of morbid changes in the body)

3) Sara pariksha (Status of elemental tissues and mind)

4) Samhanan pariksha (Compactness of the body)

5) Satva pariksha (mental strength)

6) Satmya pariksha (compatibilities)

7) Pramana pariksha (measurement of body or body parts)

8) Ahara shakti (metabolism and digestion capacity)

9) Vyayama shakti (Exercise tolerance of the patient)

10) Vaya (age)

Among all these, sara pariksha plays an important role to know the bala pramana (magnitude of physical strength) of the patient ${ }^{4}$. Sara literally means essence of dhatu, possessing all good qualities in excellent standard without any defects. The dhatus which has such an excellence will have the capacity to resist diseases and do all its normal functions efficiently. On the basis of sarata, a physician can determine as well as diagnose the bala of the rogi which will help him in treating the disease in a better way. Patients are to be examined with reference to sara or the excellence of their dhatus. With a view to determine the specific measure of bala, they are classified into eight categories ${ }^{5}$, depending upon the sara or excellence of their dhatus.
- $\quad$ Tvak sara
- Rakta sara
- $\quad$ Mamsa sara
- $\quad$ Meda sara
- $\quad$ Asthi sara
- $\quad$ Majja sara
- $\quad$ Shukra sara
- $\quad$ Satva sara

The person endowed with all the sara is sure to earn great respect, hopeful of success in all his activities, capable of withstanding troubles, will be wise and ready.

In today's era of industrialization, advanced techniques and research methods are applied for better economic gains. Due to this competition everyone is struggling to stay in the race which is automatically reducing one's attention of maintaining a good health. People are working day and night for their livelihood and neglecting the factors like ahara, nidra etc. in proper quantity and quality which have affected their life as well as health in the form of various lifestyle disorders now a days.

The present study was done to study the effect of nidra on dhatusarata which help in understanding the changes that occur on the sarata of the person when he gets altered or abnormal nidra.

\section{AIMS AND OBJECTIVES}

The study has been aimed to observe the effect of atiyoga (abnormally high), mithyayoga (improper) and heenayoga (abnormally low) of nidra on dhatusarata of willing volunteers selected for the study. 
Dr. Manisha Sharma ${ }^{1}$,International Journal of Ayurvedic \& Herbal Medicine 9(2) Mar.-Apr. 2019 (3452-3463)

\section{MATERIALS AND METHODS}

Type of study Retrospective type of study

Clinical survey

Place of study Bharati Vidyapeeth University College of Ayurveda and Bharati Vidyapeeth Medical Foundation's Ayurveda Hospital, Pune - 43.

Research Design Special case paper proforma was prepared. A total of 127 volunteers were studied on the basis of darshan (inspection), sparshan (palpation and percussion) and prashna (questioning) pareeksha. Written consent was taken from the volunteers prior to the study.

Source of Data The data was collected by examinations of volunteers from various sectors like IT professionals, doctors, staff nurses, security guards, drivers (taxi and truck), students, housewives, etc. with the help of a specially designed questionnaire and case paper. It was found that the nidra patterns of these groups were remarkably altered and hence they were included in the study.

\section{Inclusion Criteria}

$>\quad$ Volunteers having heena, mithya and atiyoga of nidra for minimum 1 year

$>\quad$ Volunteers irrespective of sex, marital status and socio-economic class

\section{Exclusion Criteria}

$>\quad$ Volunteers suffering from prior ailments of nidra

$>\quad$ Volunteers below age 16 and above 70 years

\section{Statement of Limitation}

This study was done in the rogavigyana (pathology) department and was only associated with the diagnostic aspect of the problem and hence was not directly dealt with the remedial measures.

\section{Gradation Criteria}

The volunteers included in the study were classified on the basis of following factors:

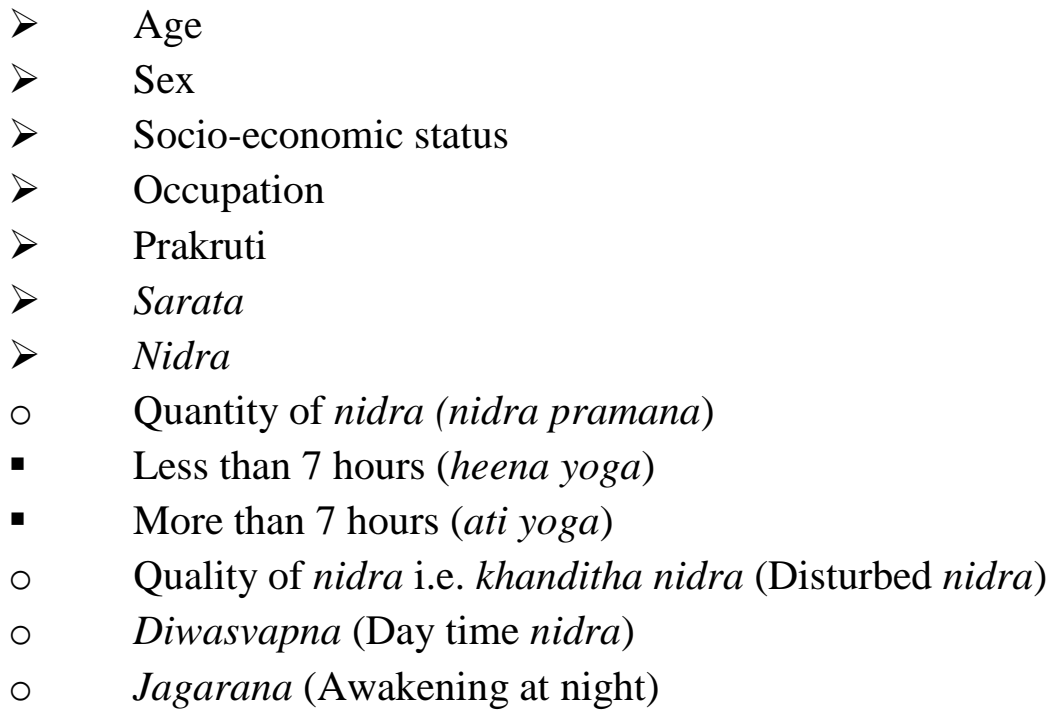

\section{Study Design}

- Initially the sarata of the individual was analyzed according to the lakshanas (symptoms) mentioned in the samhitas. The lakshanas have been graded as 0 (Aa=Absence), 1 (alpa=Mild), 2 (madhyam=Moderate), 3 (ati=Severe). Lakshanas of each sara were added separately and then from that score, percentage of each sara predominance was calculated. In this way, the percentage of all the 9 saras present in all 127 volunteers was obtained.

- Gradation of volunteers according to the hetus (etiology) like jagarana, diwasvapna, khandita nidra, less than 7 hours nidra, more than 7 hours nidra was done.

- Volunteers were separated according to the gradation criteria of each hetu related to nidra. 
Dr. Manisha Sharma ${ }^{1}$,International Journal of Ayurvedic \& Herbal Medicine 9(2) Mar.-Apr. 2019 (3452-3463) been calculated.

- $\quad$ The average of percentage of sarata in each separated group of hetus related to nidra has OBSERVATIONS AND RESULTS

Table No.1. Classification of volunteers on following factors

\begin{tabular}{|c|c|c|}
\hline Age & No.of volunteers(n) & Percentage \\
\hline $5-15$ yrs. & 1 & 0.787 \\
\hline 15-25yrs. & 30 & 23.622 \\
\hline 25-35yrs. & 49 & 38.583 \\
\hline $35-45 \mathrm{yrs}$. & 24 & 18.898 \\
\hline 45-55yrs. & 14 & 11.024 \\
\hline 55-65yrs. & 7 & 5.512 \\
\hline $65-75 y$ yrs. & 2 & 1.575 \\
\hline \multicolumn{3}{|l|}{ Sex } \\
\hline Males & 88 & 69.291 \\
\hline Females & 39 & 30.709 \\
\hline \multicolumn{3}{|l|}{ Occupation } \\
\hline Security Guard & 14 & 11.024 \\
\hline Staff Nurse & 09 & 7.087 \\
\hline Drivers & 21 & 16.535 \\
\hline Doctors & 07 & 5.512 \\
\hline IT Professionals & 45 & 35.433 \\
\hline Students & 13 & 10.236 \\
\hline Housewives & 07 & 5.512 \\
\hline Retired & 01 & 0.787 \\
\hline Others & 10 & 7.874 \\
\hline \multicolumn{3}{|l|}{ Prakriti } \\
\hline Vata Pittaja & 22 & 17.232 \\
\hline Vata Kaphaja & 12 & 9.449 \\
\hline Pitta vataj & 25 & 19.685 \\
\hline Pitta kaphaja & 24 & 18.898 \\
\hline Kapha vataj & 16 & 12.598 \\
\hline Kapha pittaja & 28 & 22.047 \\
\hline \multicolumn{3}{|c|}{ Socio economic status } \\
\hline Lower & 49 & 38.583 \\
\hline Middle & 65 & 51.181 \\
\hline Upper & 13 & 10.236 \\
\hline
\end{tabular}

Table No.2. Classification of volunteers on following factors

Sarata (Acc. To lakshanas mentioned in Charak samhita)

\begin{tabular}{|l|l|l|}
\hline 1 (Asara) & 07 & 5.512 \\
\hline 2 (Twakasara) & 14 & 11.024 \\
\hline $3($ Raktasara $)$ & 20 & 15.748 \\
\hline 4 (Mansasara $)$ & 34 & 26.772 \\
\hline
\end{tabular}


Dr. Manisha Sharma ${ }^{1}$,International Journal of Ayurvedic \& Herbal Medicine 9(2) Mar.-Apr. 2019 (3452-3463)

\begin{tabular}{|c|c|c|}
\hline 5 (Medasara) & 28 & 22.047 \\
\hline 6 (Asthisara) & 17 & 13.386 \\
\hline 7 (Majjasara) & 03 & 2.362 \\
\hline 8 (Shukrasara) & 03 & 2.362 \\
\hline 9 (Satvasara) & 01 & 0.787 \\
\hline \multicolumn{3}{|l|}{ Nidra } \\
\hline \multicolumn{3}{|l|}{ Quantity of nidra } \\
\hline \multicolumn{3}{|l|}{ - Less than 7 hrs. } \\
\hline Grade I (5-7 hrs.per day) & 30 & 14.173 \\
\hline Grade 2 (3-5 hrs.per day) & 47 & 37.008 \\
\hline Grade 3 (less than 3 hrs. per day) & 18 & 23.622 \\
\hline \multicolumn{3}{|l|}{ - More than 7 hrs. } \\
\hline Grade 1 (8-10 hrs.per day) & 06 & 6.299 \\
\hline Grade 2 (10-12 hrs.per day) & 18 & 14.173 \\
\hline Grade 3 (more than 12 hrs. per day) & 08 & 4.724 \\
\hline \multicolumn{3}{|l|}{ Quality of nidra } \\
\hline Mild khandita nidra & 61 & 48.031 \\
\hline Moderate khandita nidra & 36 & 28.346 \\
\hline Excessive khandita nidra & 30 & 23.622 \\
\hline \multicolumn{3}{|l|}{ Diwasvapna (day hrs. sleep) } \\
\hline Up to $1 \mathrm{hr}$. & 41 & 32.283 \\
\hline 2 to 3 hrs. & 64 & 50.394 \\
\hline More than $3 \mathrm{hrs}$. & 22 & 17.323 \\
\hline \multicolumn{3}{|l|}{ Jagarana (Awakening at night) } \\
\hline From $10.00 \mathrm{PM}$ to $12.00 \mathrm{AM}$ & 15 & 11.811 \\
\hline From $12.00 \mathrm{AM}$ to $2.00 \mathrm{AM}$ & 92 & 72.441 \\
\hline Above $2.00 \mathrm{AM}$ & 20 & 15.478 \\
\hline
\end{tabular}

Table no.3. Percentage of each Sara in all 127 volunteers

\begin{tabular}{|c|c|c|c|c|c|c|c|c|c|c|}
\hline & 1 & 2 & 3 & 4 & 5 & 6 & 7 & 8 & 9 & 10 \\
\hline Rasa & 51.852 & 44.444 & 48.148 & 44.444 & 18.519 & 48.148 & 48.148 & 33.333 & 44.444 & 48.148 \\
\hline Rakta & 49.020 & 68.627 & 66.667 & 37.255 & 15.686 & 31.373 & 25.490 & 45.098 & 50.980 & 49.020 \\
\hline Mansa & 66.667 & 74.074 & 51.852 & 68.519 & 3.704 & 14.815 & 33.333 & 70.370 & 87.037 & 94.444 \\
\hline Meda & 81.250 & 70.833 & 27.083 & 79.167 & 2.083 & 43.750 & 45.833 & 50.000 & 52.083 & 62.500 \\
\hline Asthi & 65.000 & 86.667 & 48.333 & 53.333 & 10.000 & 23.333 & 25.000 & 78.333 & 43.333 & 66.667 \\
\hline Majja & 58.333 & 70.833 & 41.667 & 66.667 & 0.000 & 33.333 & 50.000 & 58.333 & 50.000 & 70.833 \\
\hline Shukra & 64.286 & 69.048 & 52.381 & 23.810 & 0.000 & 21.429 & 30.952 & 57.143 & 64.286 & 66.667 \\
\hline Sattva & 66.667 & 61.111 & 69.444 & 52.778 & 61.111 & 19.444 & 52.778 & 41.667 & 47.222 & 66.667 \\
\hline Sarva & 61.111 & 66.667 & 50.000 & 50.000 & 38.889 & 11.111 & 66.667 & 44.444 & 61.111 & 72.222 \\
\hline & 11 & 12 & 13 & 14 & 15 & 16 & 17 & 18 & 19 & 20 \\
\hline Rasa & 44.444 & 59.259 & 14.815 & 59.259 & 40.741 & 44.444 & 40.741 & 51.852 & 51.852 & 25.926 \\
\hline Rakta & 60.784 & 41.176 & 15.686 & 60.784 & 54.902 & 64.706 & 72.549 & 49.020 & 78.431 & 41.176 \\
\hline Mansa & 66.667 & 38.889 & 53.704 & 98.148 & 87.037 & 88.889 & 50.000 & 66.667 & 64.815 & 42.593 \\
\hline
\end{tabular}


Dr. Manisha Sharma ${ }^{1}$,International Journal of Ayurvedic \& Herbal Medicine 9(2) Mar.-Apr. 2019 (3452-3463)

\begin{tabular}{|l|l|l|l|l|l|l|l|l|l|l|} 
Meda & 85.417 & 33.333 & 18.750 & 81.250 & 64.583 & 72.917 & 52.083 & 85.417 & 66.667 & 68.750 \\
\hline Asthi & 53.333 & 81.667 & 35.000 & 71.667 & 66.667 & 66.667 & 46.667 & 65.000 & 61.667 & 43.333 \\
\hline Majja & 45.833 & 20.833 & 8.333 & 79.167 & 70.833 & 79.167 & 58.333 & 70.833 & 62.500 & 54.167 \\
\hline Shukra & 66.667 & 33.333 & 11.905 & 66.667 & 71.429 & 66.667 & 66.667 & 59.524 & 66.667 & 66.667 \\
\hline Sattva & 38.889 & 55.556 & 13.889 & 66.667 & 77.778 & 91.667 & 66.667 & 77.778 & 50.000 & 55.556 \\
\hline Sarva & 33.333 & 50.000 & 22.222 & 77.778 & 55.556 & 61.111 & 55.556 & 61.111 & 50.000 & 61.111 \\
\hline
\end{tabular}

\begin{tabular}{|l|l|l|l|l|l|l|l|l|l|l|}
\hline & 21 & 22 & 23 & 24 & 25 & 26 & 27 & 28 & 29 & 30 \\
\hline Rasa & 44.444 & 37.037 & 55.556 & 55.556 & 11.111 & 22.222 & 37.037 & 48.148 & 3.704 & 51.852 \\
\hline Rakta & 66.667 & 68.627 & 17.647 & 9.804 & 43.137 & 66.667 & 45.098 & 45.098 & 37.255 & 41.176 \\
\hline Mansa & 68.519 & 77.778 & 42.593 & 7.407 & 16.667 & 83.333 & 66.667 & 46.296 & 61.111 & 77.778 \\
\hline Meda & 33.333 & 97.917 & 52.083 & 12.500 & 10.417 & 68.750 & 66.667 & 25.000 & 33.333 & 45.833 \\
\hline Asthi & 95.000 & 43.333 & 23.333 & 13.333 & 41.667 & 66.667 & 66.667 & 18.333 & 61.667 & 43.333 \\
\hline Majja & 66.667 & 62.500 & 33.333 & 8.333 & 4.167 & 62.500 & 33.333 & 25.000 & 33.333 & 45.833 \\
\hline Shukra & 61.905 & 66.667 & 38.095 & 4.762 & 16.667 & 66.667 & 66.667 & 33.333 & 35.714 & 57.143 \\
\hline Sattva & 52.778 & 91.667 & 33.333 & 8.333 & 8.333 & 69.444 & 66.667 & 47.222 & 77.778 & 72.222 \\
\hline Sarva & 44.444 & 61.111 & 33.333 & 22.222 & 5.556 & 61.111 & 61.111 & 50.000 & 44.444 & 38.889 \\
\hline
\end{tabular}

\begin{tabular}{|l|l|l|l|l|l|l|l|l|l|l|}
\hline & 31 & 32 & 33 & 34 & 35 & 36 & 37 & 38 & 39 & 40 \\
\hline Rasa & 55.556 & 55.556 & 29.630 & 25.926 & 62.963 & 25.926 & 44.444 & 62.963 & 48.148 & 44.444 \\
\hline Rakta & 80.392 & 54.902 & 33.333 & 47.059 & 62.745 & 33.333 & 66.667 & 100.000 & 66.667 & 56.863 \\
\hline Mansa & 72.222 & 48.148 & 66.667 & 55.556 & 100.000 & 33.333 & 66.667 & 66.667 & 100.000 & 38.889 \\
\hline Meda & 58.333 & 60.417 & 33.333 & 39.583 & 52.083 & 62.500 & 100.000 & 43.750 & 66.667 & 33.333 \\
\hline Asthi & 51.667 & 66.667 & 88.333 & 66.667 & 40.000 & 33.333 & 66.667 & 66.667 & 66.667 & 100.000 \\
\hline Majja & 33.333 & 75.000 & 33.333 & 45.833 & 33.333 & 12.500 & 33.333 & 33.333 & 33.333 & 33.333 \\
\hline Shukra & 66.667 & 66.667 & 66.667 & 66.667 & 66.667 & 66.667 & 66.667 & 66.667 & 66.667 & 66.667 \\
\hline Sattva & 66.667 & 55.556 & 66.667 & 66.667 & 66.667 & 33.333 & 66.667 & 66.667 & 66.667 & 66.667 \\
\hline Sarva & 55.556 & 66.667 & 55.556 & 72.222 & 50.000 & 22.222 & 61.111 & 61.111 & 61.111 & 66.667 \\
\hline & 41 & 42 & 43 & 44 & 45 & 46 & 47 & 48 & 49 & 50 \\
\hline Rasa & 33.333 & 55.556 & 44.444 & 48.148 & 18.519 & 48.148 & 29.630 & 55.556 & 55.556 & 70.370 \\
\hline & & 100.00 & & & & & & & & \\
Rakta & 66.667 & 0 & 88.235 & 66.667 & 33.333 & 54.902 & 66.667 & 66.667 & 100.000 & 66.667 \\
\hline & & & & 100.00 & & & & & & \\
Mansa & 74.074 & 66.667 & 66.667 & 0 & 94.444 & 62.963 & 66.667 & 66.667 & 77.778 & 66.667 \\
\hline Meda & 93.750 & 66.667 & 66.667 & 33.333 & 60.417 & 83.333 & 66.667 & 100.000 & 66.667 & 100.000 \\
\hline Asthi & 55.000 & 33.333 & 40.000 & 66.667 & 46.667 & 46.667 & 100.000 & 66.667 & 35.000 & 66.667 \\
\hline Majja & 33.333 & 33.333 & 33.333 & 33.333 & 66.667 & 33.333 & 66.667 & 41.667 & 66.667 & 33.333 \\
\hline Shukra & 66.667 & 66.667 & 66.667 & 66.667 & 66.667 & 66.667 & 66.667 & 66.667 & 66.667 & 66.667 \\
\hline Sattva & 66.667 & 66.667 & 66.667 & 66.667 & 66.667 & 66.667 & 66.667 & 66.667 & 52.778 & 66.667 \\
\hline Sarva & 61.111 & 66.667 & 50.000 & 61.111 & 50.000 & 50.000 & 66.667 & 50.000 & 55.556 & 61.111 \\
\hline & & & & & & & & & & \\
\hline
\end{tabular}

\begin{tabular}{|l|l|l|l|l|l|l|l|l|l|l|}
\hline & 51 & 52 & 53 & 54 & 55 & 56 & 57 & 58 & 59 & 60 \\
\hline Rasa & 37.037 & 48.148 & 44.444 & 33.333 & 33.333 & 22.222 & 11.111 & 14.815 & 11.111 & 11.111 \\
\hline Rakta & 33.333 & 54.902 & 33.333 & 39.216 & 52.941 & 11.765 & 23.529 & 17.647 & 19.608 & 15.686 \\
\hline Mansa & 66.667 & 42.593 & 61.111 & 18.519 & 66.667 & 14.815 & 29.630 & 24.074 & 16.667 & 11.111 \\
\hline
\end{tabular}


Dr. Manisha Sharma ${ }^{1}$,International Journal of Ayurvedic \& Herbal Medicine 9(2) Mar.-Apr. 2019 (3452-3463)

\begin{tabular}{|l|l|l|l|l|l|l|l|l|l|l|} 
Meda & 33.333 & 62.500 & 37.500 & 12.500 & 75.000 & 18.750 & 20.833 & 12.500 & 14.583 & 12.500 \\
\hline Asthi & 66.667 & 66.667 & 53.333 & 20.000 & 66.667 & 16.667 & 20.000 & 26.667 & 15.000 & 45.000 \\
\hline Majja & 33.333 & 33.333 & 33.333 & 16.667 & 33.333 & 16.667 & 8.333 & 25.000 & 12.500 & 16.667 \\
\hline Shukra & 66.667 & 66.667 & 66.667 & 21.429 & 54.762 & 21.429 & 40.476 & 19.048 & 14.286 & 21.429 \\
\hline Sattva & 33.333 & 36.111 & 33.333 & 27.778 & 55.556 & 19.444 & 25.000 & 25.000 & 13.889 & 16.667 \\
\hline Sarva & 38.889 & 44.444 & 44.444 & 22.222 & 33.333 & 16.667 & 5.556 & 11.111 & 27.778 & 5.556 \\
\hline
\end{tabular}

\begin{tabular}{|l|l|l|l|l|l|l|l|l|l|l|}
\hline & 61 & 62 & 63 & 64 & 65 & 66 & 67 & 68 & 69 & 70 \\
\hline Rasa & 33.333 & 33.333 & 55.556 & 22.222 & 81.481 & 25.926 & 74.074 & 44.444 & 40.741 & 66.667 \\
\hline Rakta & 45.098 & 54.902 & 58.824 & 25.490 & 15.686 & 43.137 & 15.686 & 7.843 & 58.824 & 25.490 \\
\hline Mansa & 57.407 & 42.593 & 55.556 & 48.148 & 50.000 & 18.519 & 24.074 & 14.815 & 51.852 & 44.444 \\
\hline Meda & 54.167 & 54.167 & 54.167 & 52.083 & 12.500 & 27.083 & 45.833 & 10.417 & 43.750 & 43.750 \\
\hline Asthi & 50.000 & 60.000 & 40.000 & 20.000 & 18.333 & 61.667 & 20.000 & 53.333 & 31.667 & 63.333 \\
\hline Majja & 33.333 & 33.333 & 33.333 & 8.333 & 16.667 & 16.667 & 8.333 & 20.833 & 16.667 & 29.167 \\
\hline Shukra & 66.667 & 33.333 & 66.667 & 14.286 & 16.667 & 23.810 & 9.524 & 16.667 & 42.857 & 61.905 \\
\hline Sattva & 44.444 & 50.000 & 50.000 & 16.667 & 13.889 & 19.444 & 13.889 & 22.222 & 25.000 & 33.333 \\
\hline Sarva & 33.333 & 66.667 & 61.111 & 22.222 & 5.556 & 16.667 & 5.556 & 16.667 & 33.333 & 61.111 \\
\hline
\end{tabular}

\begin{tabular}{|l|l|l|l|l|l|l|l|l|l|l|}
\hline & 71 & 72 & 73 & 74 & 75 & 76 & 77 & 78 & 79 & 80 \\
\hline Rasa & 44.444 & 37.037 & 48.148 & 66.667 & 37.037 & 25.926 & 55.556 & 44.444 & 62.963 & 62.963 \\
\hline Rakta & 33.333 & 43.137 & 54.902 & 66.667 & 54.902 & 66.667 & 54.902 & 39.216 & 62.745 & 66.667 \\
\hline Mansa & 42.593 & 66.667 & 61.111 & 94.444 & 77.778 & 33.333 & 66.667 & 62.963 & 66.667 & 66.667 \\
\hline Meda & 37.500 & 81.250 & 58.333 & 56.250 & 79.167 & 0.000 & 66.667 & 66.667 & 66.667 & 47.917 \\
\hline Asthi & 46.667 & 48.333 & 66.667 & 55.000 & 73.333 & 33.333 & 41.667 & 66.667 & 66.667 & 66.667 \\
\hline Majja & 33.333 & 33.333 & 33.333 & 33.333 & 33.333 & 0.000 & 33.333 & 33.333 & 33.333 & 37.500 \\
\hline Shukra & 40.476 & 66.667 & 66.667 & 66.667 & 33.333 & 33.333 & 66.667 & 33.333 & 33.333 & 33.333 \\
\hline Sattva & 27.778 & 52.778 & 50.000 & 50.000 & 66.667 & 55.556 & 50.000 & 33.333 & 66.667 & 91.667 \\
\hline Sarva & 38.889 & 55.556 & 55.556 & 66.667 & 72.222 & 33.333 & 66.667 & 33.333 & 66.667 & 44.444 \\
\hline
\end{tabular}

\begin{tabular}{|l|l|l|l|l|l|l|l|l|l|l|}
\hline & 81 & 82 & 83 & 84 & 85 & 86 & 87 & 88 & 89 & 90 \\
\hline Rasa & 14.815 & 55.556 & 51.852 & 55.556 & 55.556 & 33.333 & 37.037 & 37.037 & 37.037 & 51.852 \\
\hline Rakta & 17.647 & 54.902 & 49.020 & 43.137 & 45.098 & 31.373 & 49.020 & 41.176 & 45.098 & 45.098 \\
\hline Mansa & 14.815 & 57.407 & 68.519 & 68.519 & 50.000 & 25.926 & 59.259 & 51.852 & 59.259 & 62.963 \\
\hline Meda & 45.833 & 47.917 & 50.000 & 50.000 & 43.750 & 20.833 & 43.750 & 33.333 & 62.500 & 50.000 \\
\hline Asthi & 40.000 & 51.667 & 41.667 & 51.667 & 55.000 & 60.000 & 50.000 & 46.667 & 48.333 & 55.000 \\
\hline Majja & 45.833 & 33.333 & 33.333 & 20.833 & 54.167 & 37.500 & 33.333 & 33.333 & 33.333 & 37.500 \\
\hline Shukra & 42.857 & 33.333 & 66.667 & 52.381 & 69.048 & 33.333 & 66.667 & 66.667 & 59.524 & 66.667 \\
\hline Sattva & 27.778 & 63.889 & 86.111 & 58.333 & 50.000 & 61.111 & 61.111 & 52.778 & 50.000 & 50.000 \\
\hline Sarva & 38.889 & 44.444 & 61.111 & 44.444 & 50.000 & 55.556 & 38.889 & 38.889 & 33.333 & 44.444 \\
\hline
\end{tabular}

\begin{tabular}{|l|l|l|l|l|l|l|l|l|l|l|}
\hline & 91 & 92 & 93 & 94 & 95 & 96 & 97 & 98 & 99 & 100 \\
\hline Rasa & 37.037 & 51.852 & 48.148 & 48.148 & 44.444 & 59.259 & 33.333 & 25.926 & 25.926 & 29.630 \\
\hline Rakta & 45.098 & 39.216 & 54.902 & 33.333 & 58.824 & 88.235 & 33.333 & 49.020 & 33.333 & 7.843 \\
\hline Mansa & 48.148 & 48.148 & 68.519 & 33.333 & 66.667 & 66.667 & 55.556 & 37.037 & 33.333 & 12.963 \\
\hline Meda & 20.833 & 39.583 & 60.417 & 33.333 & 66.667 & 66.667 & 62.500 & 16.667 & 0.000 & 22.917 \\
\hline
\end{tabular}


Dr. Manisha Sharma ${ }^{1}$,International Journal of Ayurvedic \& Herbal Medicine 9(2) Mar.-Apr. 2019 (3452-3463)

\begin{tabular}{|l|l|l|l|l|l|l|l|l|l|l|} 
Asthi & 45.000 & 70.000 & 66.667 & 33.333 & 66.667 & 90.000 & 33.333 & 33.333 & 33.333 & 33.333 \\
\hline Majja & 20.833 & 29.167 & 58.333 & 33.333 & 33.333 & 66.667 & 33.333 & 25.000 & 0.000 & 0.000 \\
\hline Shukra & 50.000 & 66.667 & 66.667 & 33.333 & 33.333 & 66.667 & 33.333 & 33.333 & 33.333 & 0.000 \\
\hline Sattva & 47.222 & 44.444 & 52.778 & 50.000 & 66.667 & 66.667 & 33.333 & 44.444 & 11.111 & 55.556 \\
\hline Sarva & 50.000 & 50.000 & 55.556 & 38.889 & 61.111 & 66.667 & 55.556 & 44.444 & 16.667 & 16.667 \\
\hline
\end{tabular}

\begin{tabular}{|l|l|l|l|l|l|l|l|l|l|l|}
\hline & 101 & 102 & 103 & 104 & 105 & 106 & 107 & 108 & 109 & 110 \\
\hline Rasa & 37.037 & 29.630 & 29.630 & 37.037 & 44.444 & 66.667 & 44.444 & 55.556 & 40.741 & 59.259 \\
\hline Rakta & 39.216 & 33.333 & 74.510 & 56.863 & 64.706 & 45.098 & 39.216 & 60.784 & 47.059 & 33.333 \\
\hline Mansa & 53.704 & 53.704 & 53.704 & 79.630 & 64.815 & 55.556 & 66.667 & 72.222 & 72.222 & 31.481 \\
\hline Meda & 62.500 & 68.750 & 45.833 & 77.083 & 66.667 & 79.167 & 72.917 & 77.083 & 35.417 & 31.250 \\
\hline Asthi & 60.000 & 66.667 & 66.667 & 66.667 & 53.333 & 26.667 & 33.333 & 58.333 & 65.000 & 41.667 \\
\hline Majja & 33.333 & 25.000 & 33.333 & 16.667 & 33.333 & 33.333 & 16.667 & 62.500 & 37.500 & 33.333 \\
\hline Shukra & 33.333 & 42.857 & 66.667 & 66.667 & 100.000 & 23.810 & 42.857 & 66.667 & 66.667 & 33.333 \\
\hline Sattva & 77.778 & 61.111 & 88.889 & 44.444 & 77.778 & 38.889 & 63.889 & 61.111 & 58.333 & 47.222 \\
\hline Sarva & 55.556 & 44.444 & 38.889 & 5.556 & 61.111 & 16.667 & 38.889 & 55.556 & 66.667 & 22.222 \\
\hline
\end{tabular}

\begin{tabular}{|l|l|l|l|l|l|l|l|l|l|l|}
\hline & 111 & 112 & 113 & 114 & 115 & 116 & 117 & 118 & 119 & 120 \\
\hline Rasa & 55.556 & 33.333 & 51.852 & 51.852 & 25.926 & 55.556 & 59.259 & 70.370 & 70.370 & 62.963 \\
\hline Rakta & 47.059 & 33.333 & 56.863 & 60.784 & 9.804 & 74.510 & 84.314 & 66.667 & 54.902 & 45.098 \\
\hline Mansa & 42.593 & 24.074 & 38.889 & 81.481 & 12.963 & 61.111 & 75.926 & 70.370 & 75.926 & 48.148 \\
\hline Meda & 39.583 & 33.333 & 33.333 & 66.667 & 18.750 & 66.667 & 95.833 & 54.167 & 81.250 & 50.000 \\
\hline Asthi & 60.000 & 35.000 & 43.333 & 73.333 & 11.667 & 73.333 & 71.667 & 80.000 & 56.667 & 33.333 \\
\hline Majja & 33.333 & 16.667 & 4.167 & 41.667 & 12.500 & 66.667 & 25.000 & 33.333 & 33.333 & 33.333 \\
\hline Shukra & 54.762 & 45.238 & 40.476 & 66.667 & 19.048 & 61.905 & 66.667 & 66.667 & 54.762 & 45.238 \\
\hline Sattva & 61.111 & 33.333 & 55.556 & 83.333 & 30.556 & 66.667 & 52.778 & 86.111 & 44.444 & 86.111 \\
\hline Sarva & 44.444 & 16.667 & 50.000 & 72.222 & 11.111 & 77.778 & 66.667 & 72.222 & 38.889 & 61.111 \\
\hline
\end{tabular}

\begin{tabular}{|l|l|l|l|l|l|l|l|}
\hline & 121 & 122 & 123 & 124 & 125 & 126 & 127 \\
\hline Rasa & 66.667 & 59.259 & 51.852 & 77.778 & 48.148 & 40.741 & 59.259 \\
\hline Rakta & 76.471 & 49.020 & 47.059 & 62.745 & 64.706 & 33.333 & 45.098 \\
\hline Mansa & 70.370 & 66.667 & 38.889 & 48.148 & 64.815 & 27.778 & 66.667 \\
\hline Meda & 75.000 & 50.000 & 33.333 & 33.333 & 70.833 & 66.667 & 33.333 \\
\hline Asthi & 85.000 & 60.000 & 53.333 & 73.333 & 41.667 & 40.000 & 50.000 \\
\hline Majja & 33.333 & 20.833 & 16.667 & 29.167 & 25.000 & 33.333 & 33.333 \\
\hline Shukra & 66.667 & 66.667 & 33.333 & 61.905 & 78.571 & 50.000 & 47.619 \\
\hline Sattva & 88.889 & 61.111 & 44.444 & 66.667 & 75.000 & 63.889 & 88.889 \\
\hline Sarva & 61.111 & 50.000 & 44.444 & 61.111 & 61.111 & 44.444 & 55.556 \\
\hline
\end{tabular}

The above tables represent the Percentage of each Sara in all the 127 volunteers.

\section{Effect of different parameters of nidra on dhatusarata}

$>$ Less than 7 hours of nidra 


\section{Graph no.1}

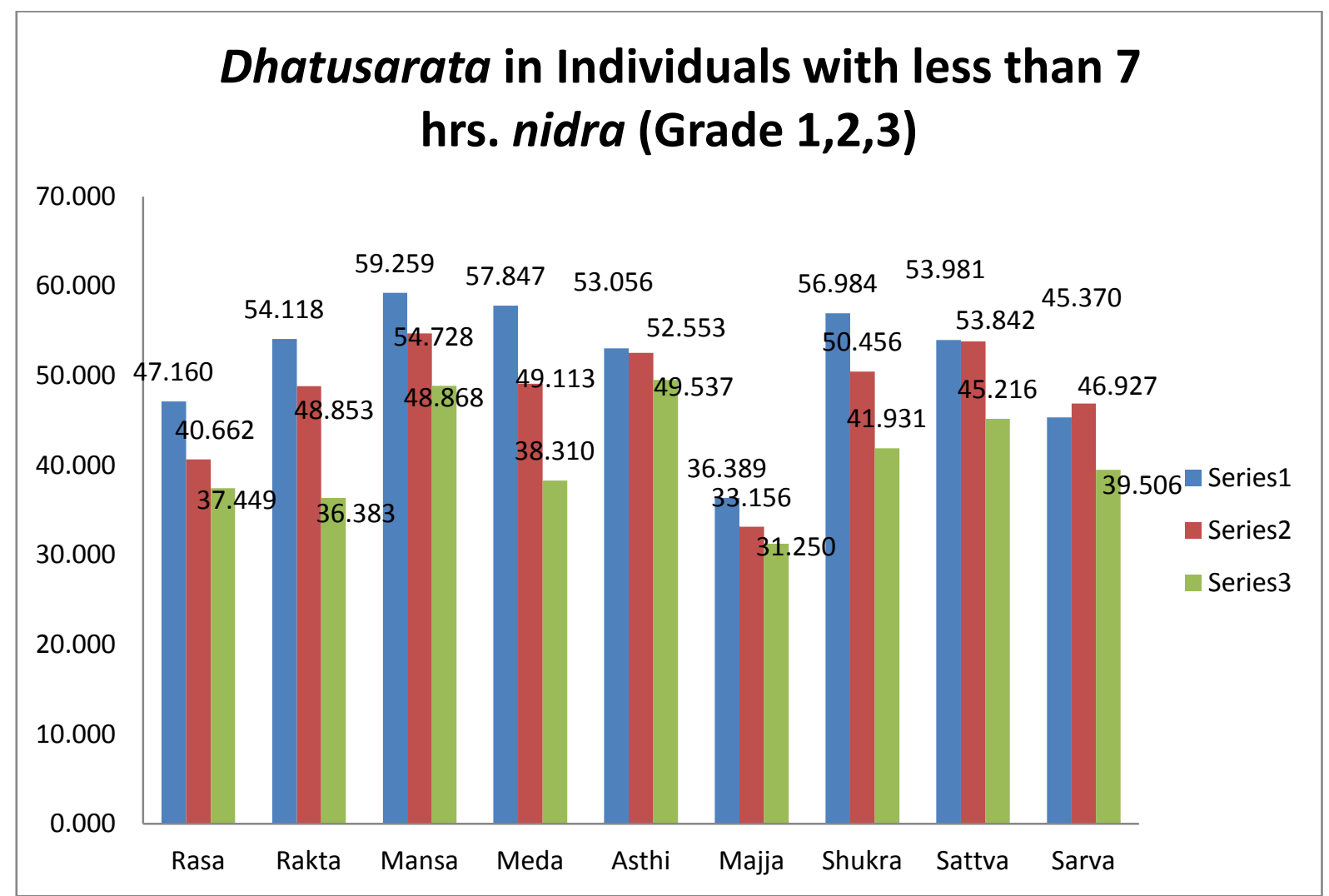

The above graph reveals that as the number of hours of nidra was reduced (heena yoga of nidra), average of each sara also got affected. The impact was drastically noted on mamsasara, followed by medasara and then by shukrasara through the first gradation criteria i.e nidra for 5-7 hours. The same sara was involved in second gradation criteria (3-5 hours nidra). But in case of third gradation criteria ( $<3$ hours), it was observed that majjasara was affected the most followed by rakta and then by rasasara.

$>$ More than 7 hours of nidra

\section{Graph no.2}


Dr. Manisha Sharma ${ }^{1}$,International Journal of Ayurvedic \& Herbal Medicine 9(2) Mar.-Apr. 2019 (3452-3463)

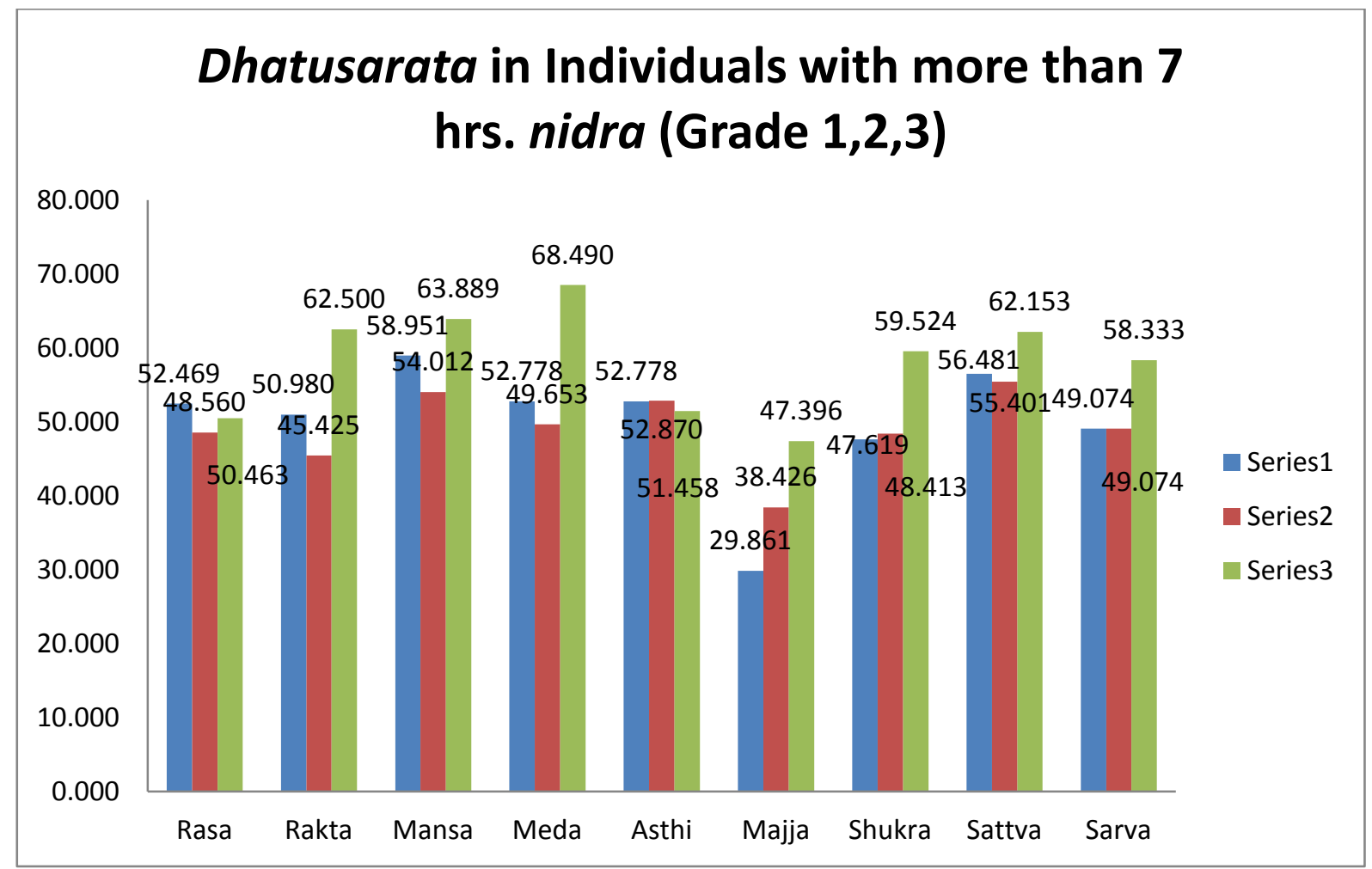

The above chart reveals that as the number of hours of nidra was increased (ati yoga of nidra), average of each sara also got increased. As the number of hours of nidra were increased, medasara was affected the most followed by satvasara and by mamsasara.

\section{Khandita nidra}

\section{Graph no.3}

\section{Effect of khandita nidra in dhatusarata}

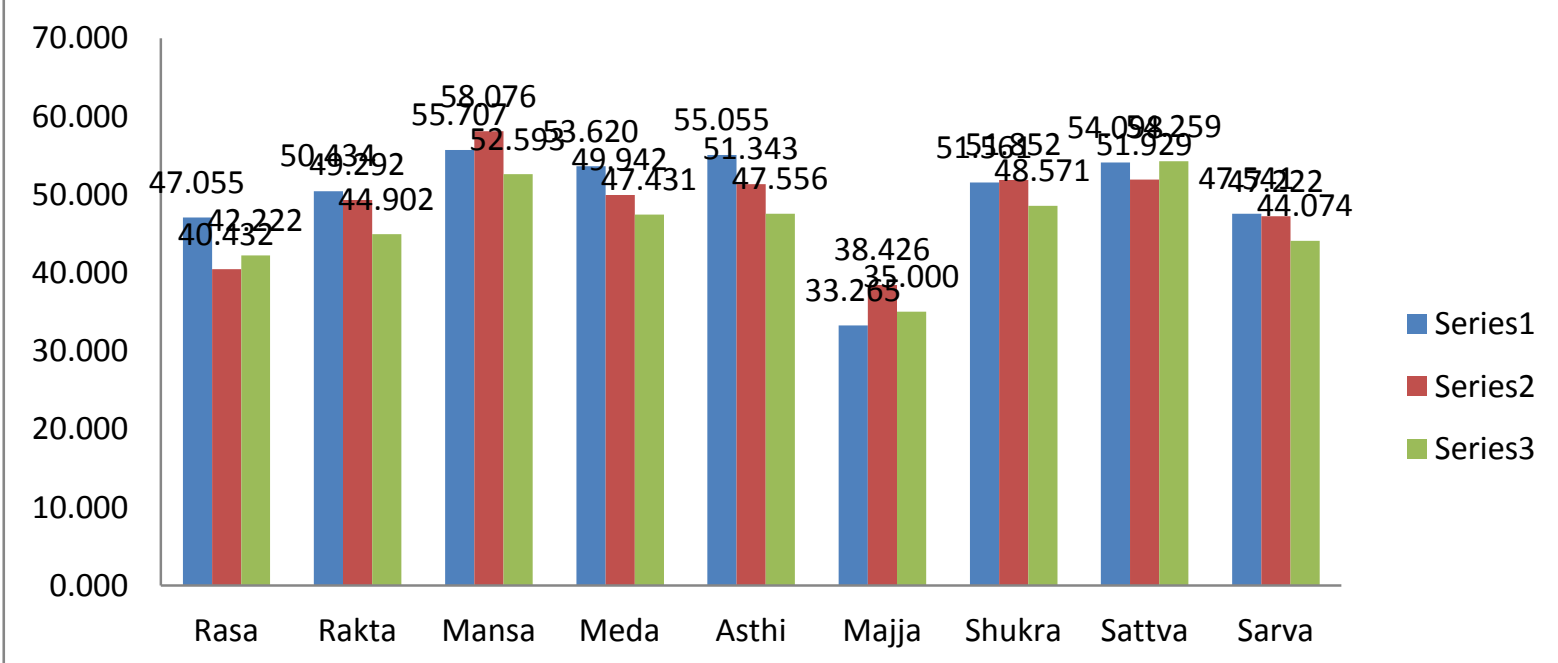

As the nidra quality i.e khandita nidra increases, sarata gets affected. This can be properly understood by seeing the graphs of rasa, meda, asthi, shukra and sarvasara.

$>$ Diwasvapna

Graph no.4 
Dr. Manisha Sharma ${ }^{1}$,International Journal of Ayurvedic \& Herbal Medicine 9(2) Mar.-Apr. 2019 (3452-3463)

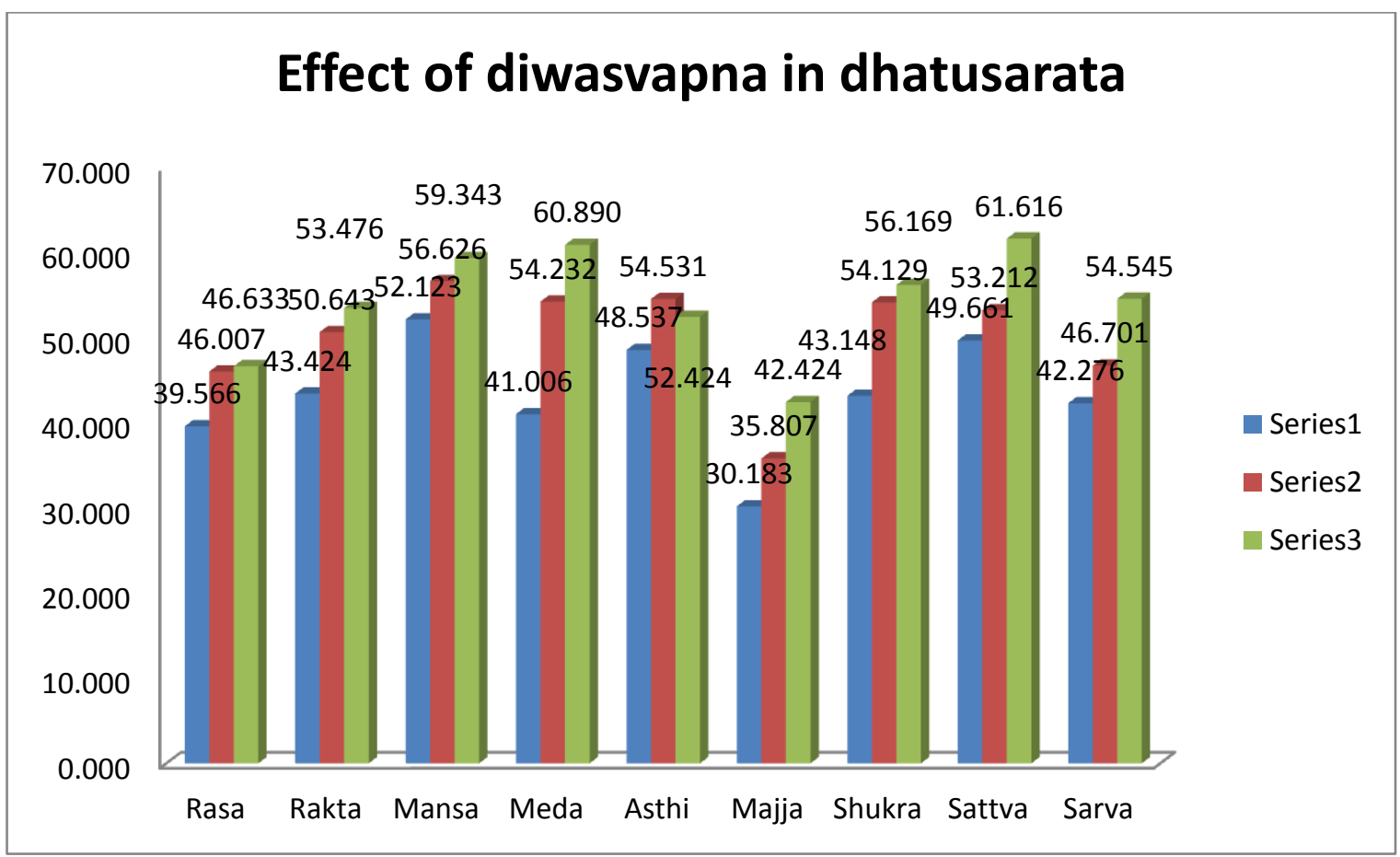

In this graph, as the nidra increased in day hours, sarata got affected. Significant change was seen in satvasara followed by medasara followed by mamsasara which went along with the principles mentioned in the samhitas that nidra in daytime affected the satva of a person and increased the meda dhatu.

$>$ Jagrana

Graph no.5

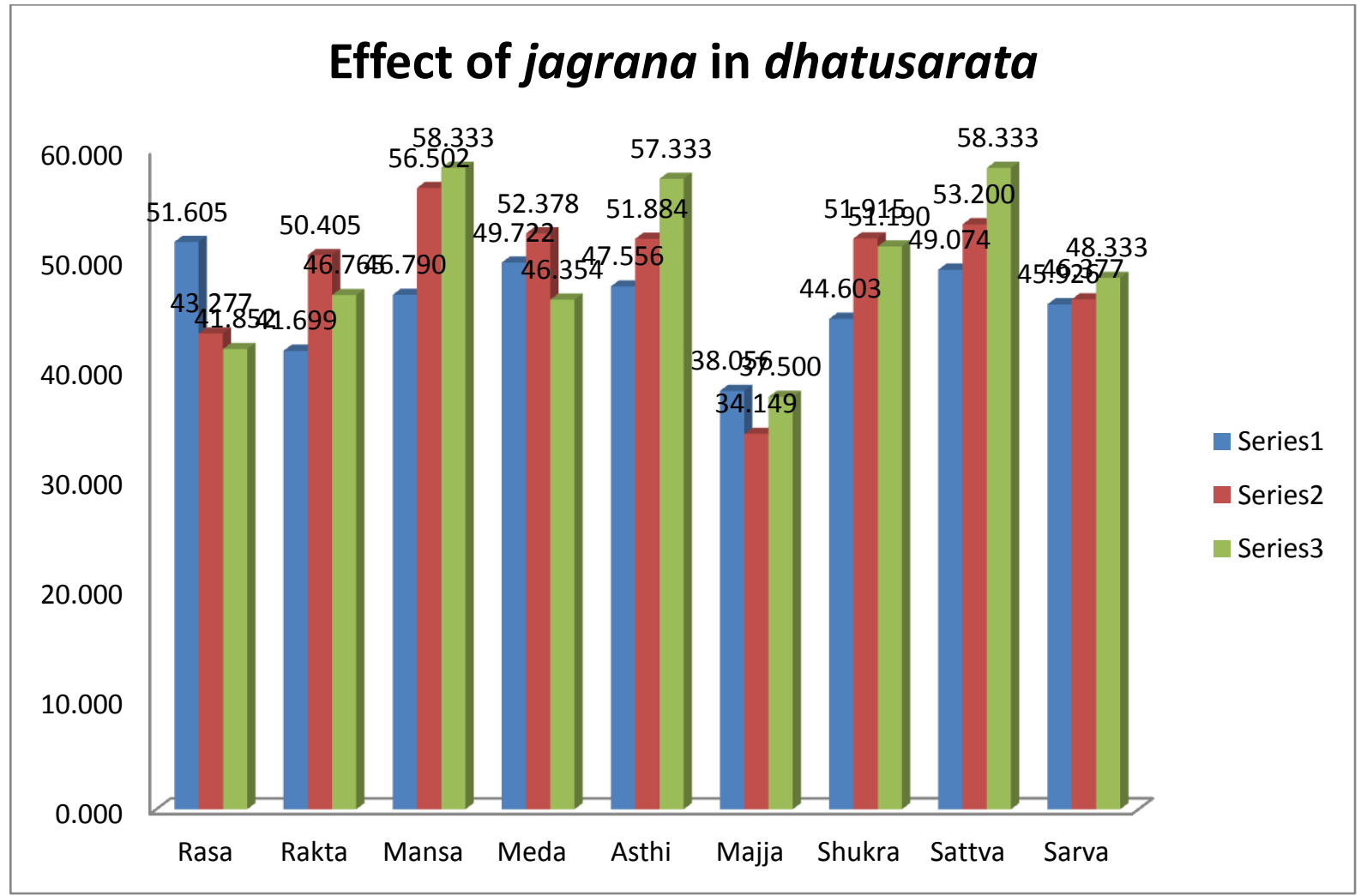

It was seen that as jagrana was increased, sarata was getting affected. Though a specific conclusion could not be made out from this graph but rasasara, medasara and majja sara were showing the results in our favour. It was found that in all these three saras, average of the first grade was much better than the average of the third grade which proves that as the number of hours of awakening increase, sarata gets affected. 
Dr. Manisha Sharma ${ }^{1}$,International Journal of Ayurvedic \& Herbal Medicine 9(2) Mar.-Apr. 2019 (3452-3463)

\section{CONCLUSION}

It was concluded from the study that-

- Nidra and dhatusarata are closely associated.

- Every form of nidra viparyaya affects sarata of one dhatu or the other, thus making it one of the major hetus for vyadhis.

- Rasadhatu sarata is affected in every form of nidra viparyaya. This is highly significant as it is likely to affect other dhatus as well.

- In every form, as the severity of nidra viparyaya increases, the severity of disturbance in dhatusarata also increases.

- Heena yoga of nidra affects rasa, asthi and satvasarata more than other dhatus.

- Ati yoga of nidra leads to nourishment of snigdha dhatus, especially mamsa, meda, rakta and rasa. The trend of increasing dhatus would continue into dhatu vruddhi as the severity of ati-nidra increase.

- In mithya yoga

- Khandita nidra leads to variation in asthi, rasa, rakta and majjasara.

- Diwasvapna is a known cause of kapha and pitta prakopa which leads to meda, majja, shukra and satvasara vitiation.

Jagarana has been found to be affecting the rasa, majja, asthi and satvasarata significantly. It also leads to sarva dhatu kshaya.

This paper is an attempt to show the correlation between dhatusarata and hetus related to nidra but there are some other factors, apart from hetus related to nidra which affects dhatusarata like ahara vidhi i.e.samashana, adhyashana, vishamashana etc.; guna sevana i.e. ruksha, snigdha, laghu etc; rasa sevana i.e.madhura, amla, lavana etc., type of diet i.e.vegetarian and non vegetarian diet; vihara i.e. vyayama or chankramana; vyasana i.e.coffee, tea, smoking etc; manasika hetu i.e. krodha, shoka, chinta etc. So further study is required to show the effect of sharirika and manasika bhavas on dhatusarata..

\section{REFERENCES}

1. Pt. Kashinath Pandey, Dr. Gorakhnath Chaturvedi. Charak Samhita,Vol. I, Sutrasthana, edition 2009, Chaukhambha Bharati Academy, Varanasi. Chapter 30/26, Page no. 587

2. Pt. Kashinath Pandey, Dr. Gorakhnath Chaturvedi. Charak Samhita,Vol. I, Sutrasthana, edition 2009, Chaukhambha Bharati Academy, Varanasi. Chapter 11/35, Page no. 227

3. Pt. Kashinath Pandey, Dr. Gorakhnath Chaturvedi. Charak Samhita,Vol. I, Vimanasthana, edition 2009, Chaukhambha Bharati Academy, Varanasi. Chapter 8/94, Page no. 771

4. Pt. Kashinath Pandey, Dr. Gorakhnath Chaturvedi. Charak Samhita,Vol. I, Vimanasthana, edition 2009, Chaukhambha Bharati Academy, Varanasi. Chapter 8/102, Page no. 775

5. Pt. Kashinath Pandey, Dr. Gorakhnath Chaturvedi. Charak Samhita,Vol. I, Vimanasthana, edition 2009, Chaukhambha Bharati Academy, Varanasi. Chapter 8/102, Page no. 775 